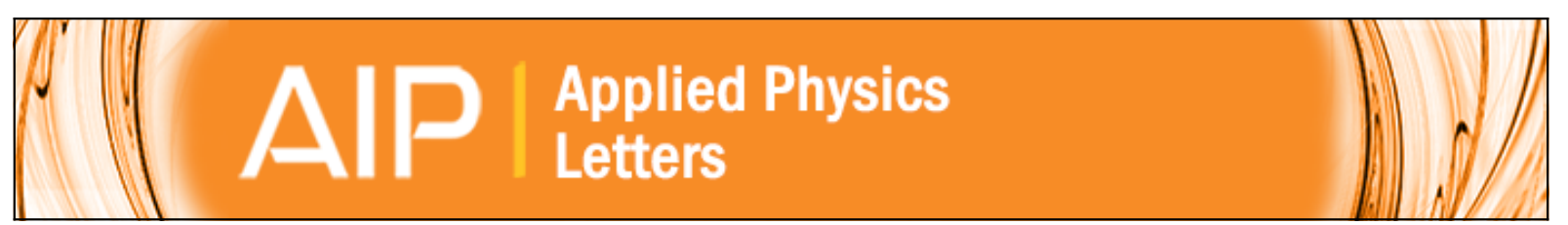

Charge carrier generation, relaxation, and recombination in polytypic GaAs nanowires studied by photoluminescence excitation spectroscopy

P. Corfdir, B. Van Hattem, E. Uccelli, A. Fontcuberta i Morral, and R. T. Phillips

Citation: Applied Physics Letters 103, 133109 (2013); doi: 10.1063/1.4822345

View online: http://dx.doi.org/10.1063/1.4822345

View Table of Contents: http://scitation.aip.org/content/aip/journal/apl/103/13?ver=pdfcov

Published by the AIP Publishing

$\underset{\substack{\text { Publoning } \\ \text { PIP }}}{A}$ Re-register for Table of Content Alerts

Create a profile.

Sign up today! 


\title{
Charge carrier generation, relaxation, and recombination in polytypic GaAs nanowires studied by photoluminescence excitation spectroscopy
}

\author{
P. Corfdir, ${ }^{1, a)}$ B. Van Hattem, ${ }^{1}$ E. Uccelli, ${ }^{2,3, b)}$ A. Fontcuberta i Morral, ${ }^{2}$ and R. T. Phillips ${ }^{1}$ \\ ${ }^{1}$ Cavendish Laboratory, University of Cambridge, J. J. Thomson Avenue, CB3 OHE Cambridge, \\ United Kingdom \\ ${ }^{2}$ Laboratoire des Matériaux Semiconducteurs, Ecole Polytechnique Fédérale de Lausanne, 1015 Lausanne, \\ Switzerland \\ ${ }^{3}$ Walter Schottky Institut and Physik Department, Technische Universität München, 85747 Garching, Germany
}

(Received 22 August 2013; accepted 10 September 2013; published online 26 September 2013)

\begin{abstract}
We report results of a study of polytypic GaAs nanowires using low-temperature photoluminescence excitation spectroscopy. The nanowire ensemble shows a strong absorption at $1.517 \mathrm{eV}$, as a result of resonant generation of heavy-hole excitons in the zinc-blende segments of the nanowires. Excitons then diffuse along the length of the nanowire and are trapped by the type-II quantum discs arising from the zinc-blende/wurtzite crystal structure alternation and recombine radiatively. Finally, experiments on single nanowires demonstrate that the energy of the $\Gamma_{7}$ conduction band to $\Gamma_{9}$ valence band exciton of wurtzite GaAs is $1.521 \mathrm{eV}$ at $4 \mathrm{~K}$.
\end{abstract}

(C) 2013 AIP Publishing LLC. [http://dx.doi.org/10.1063/1.4822345]

One-dimensional semiconductor nanostructures are currently attracting huge interest for optoelectronic applications, as they can improve efficiency of solar cells, ${ }^{1,2}$ and provide high-yield single photon emitters ${ }^{3-8}$ or low-threshold coherent light emitters. ${ }^{9,10}$ Non-nitride III-V semiconductor layers grow in a zinc-blende (ZB) crystal structure, but in the case of nanowires it is possible to grow III-V materials in a wurtzite (WZ) or a ZB form. The possibility of tuning the crystal phase along the length of nanowires offers additional degrees of freedom regarding the tuning of the electronic properties of nanowires. Not only do the WZ and ZB crystal phases of a III-V semiconductor have different electronic properties, ${ }^{12}$ but it is also now possible to realize superlattices ${ }^{12}$ or quantum dots ${ }^{13}$ based on the alternation of $\mathrm{WZ}$ and $\mathrm{ZB}$ phases along the length of nanowires. WZ GaAs nanowires have been grown in which the stacking faults are few, or entirely absent. ${ }^{14-16}$ Despite this technical progress, the band structure of WZ GaAs remains unclear. There is still controversy surrounding the value of the bandgap ${ }^{15,17-20}$ and of the electron effective mass, ${ }^{11,21-23}$ and the symmetry of the lowest energy conduction band has yet to be clarified. ${ }^{15,16,24}$ Nonresonant photoluminescence (PL) has been useful in elucidating the interplay between charge carrier relaxation and recombination mechanisms, but it cannot alone answer the questions regarding the band structure as the nanowire emission near the band-edge at low-temperature is dominated by the recombination of excitons bound to point ${ }^{25-27}$ and extended defects. ${ }^{22,28,29}$ Resonant techniques, such as photoluminescence excitation spectroscopy (PLE) ${ }^{28}$ and resonant Raman scattering (RRS), ${ }^{15,16}$ are preferable for probing the band structure of nanowires with WZ crystal structure.

In this letter, we present a combined low-temperature PLE and PL study of polytypic GaAs nanowires grown by catalyst-free molecular beam epitaxy. Based on experiments

\footnotetext{
${ }^{a)}$ Electronic mail: pmc53@cam.ac.uk

${ }^{b}$ Present address: IBM Zürich Research Laboratory, 8803 Rüschlikon, Switzerland.
}

on ensembles and on single nanowires, we describe the generation, relaxation, and recombination processes of excitons for laser excitation energy in the $1.512-1.700 \mathrm{eV}$ range. Finally, we deduce that the energy of the $\Gamma_{7}$ conduction band to $\Gamma_{9}$ valence band exciton of WZ GaAs is $1.521 \mathrm{eV}$ at $4 \mathrm{~K}$, and we discuss in terms of heavy-electron light-electron mixing the implications of this result for the electronic properties of GaAs WZ/ZB quantum discs.

GaAs nanowires have been grown by molecular beam epitaxy on $\mathrm{SiO}_{2}$-coated (111)B GaAs. During the growth, the As partial pressure was increased from $8.8 \times 10^{-7}$ to $3.3 \times 10^{-6}$ millibars. The nanowires exhibit a length of a few microns and a diameter of typically $200 \mathrm{~nm}$. The average volume fraction of material with $\mathrm{ZB}$ crystal phase is larger than $80 \%$. The two ends of the nanowires exhibit faulted ZB and WZ structures, respectively, and the intermediate segments show a non-periodic alternation between $\mathrm{WZ}$ and $\mathrm{ZB}$ phases with spacing in the range $1-10 \mathrm{~nm} \cdot{ }^{17,22}$ The nanowires were capped by an $\mathrm{Al}_{0.33} \mathrm{Ga}_{0.67} \mathrm{As}$ shell in order to reduce the efficiency of non-radiative surface recombination. ${ }^{30}$ For experiments on single nanowires, we dispersed the wires on a $\mathrm{Si}$ substrate. The ensembles and dispersed nanowire samples were held in a continuous flow He cryostat operating at $4 \mathrm{~K}$. PL and PLE experiments were carried out using a continuous-wave Ti:Sapphire laser continuously tunable between 1.4 and $1.7 \mathrm{eV}$ and with linewidth smaller than $160 \mu \mathrm{eV}$. The laser power and wavelength were monitored with an (In,Ga)As photodiode and a Fizeau interferometer, respectively. The laser light was focused with a microscope objective of NA $=0.5$ down to a $1.5 \mu \mathrm{m}$ diameter spot and the nanowire emission was collected by the same objective. For PL experiments, the signal was sent to a $64 \mathrm{~cm}$ focal length spectrograph equipped with 600 and 1200 grooves per $\mathrm{mm}$ gratings followed by a CCD. In the case of PLE experiments, the nanowire emission was sent to a $64 \mathrm{~cm}$ focal length triple spectrometer working in subtractive mode, allowing the analysis of emission energetically close to the laser line (closer than $1 \mathrm{meV}$ ). For PL and PLE experiments 
on ensembles and on single nanowires, the laser was polarized perpendicularly to the nanowires axis (i.e., polarization perpendicular to the [111] and [0001] crystallographic axes of $\mathrm{ZB}$ and $\mathrm{WZ} \mathrm{GaAs,} \mathrm{respectively).}$

We show in Figure 1(a) a PL spectrum of an ensemble of as-grown nanowires taken with excitation at $1.650 \mathrm{eV}$ and a power of $3 \mathrm{nW}$. The wire PL shows several emission peaks in the $1.440-1.515 \mathrm{eV}$ range. The number and the positions in energy of these emission lines vary from place to place on the sample and their linewidths range typically between $500 \mu \mathrm{eV}$ and a few meV. When increasing the excitation density up to $8 \mu \mathrm{W}$, the shape of the multi-peak spectrum evolves towards a Gaussian centred at $1.495 \mathrm{eV}$ with a full width at half maximum of $35 \mathrm{meV}$ (Figure 1(b)). On its highenergy side, we detect an additional emission band centred at $1.516 \mathrm{eV}$ with a $7 \mathrm{meV}$ broadening. In agreement with previous reports, ${ }^{17,22}$ we attribute the PL emission between 1.440 and $1.515 \mathrm{eV}$ to the recombination of excitons in polytypic segments of the nanowires. The narrowest lines observed here arise from the recombination of excitons bound to single WZ/ZB quantum discs. The emission energy of such excitons depends strongly on the local coupling scheme between WZ and ZB GaAs, as discussed in Refs. 22 and 31. When the excitation density is increased, filling of available quantum disc states and population of the nearly fault-free WZ and ZB segments result in a broadening of the discs PL and in the appearance of the $1.516 \mathrm{eV}$ band, respectively (Figure 1(b)). Finally, we note that for an excitation power larger than $2 \mu \mathrm{W}$, heating of the sample leads to a redshift of the nanowire PL, and analysis of the high-energy side of the $1.516 \mathrm{eV}$ PL band also indicates an increase in effective carrier temperature (from 25 to $100 \mathrm{~K}$, when the excitation power is increased from $2 \mu \mathrm{W}$ to $3 \mathrm{~mW}$ ).

We show in Figure 2(a) a typical micro-PL scan taken along the length of a single nanowire and obtained with a laser excitation at an energy of $1.650 \mathrm{eV}$. The energy of

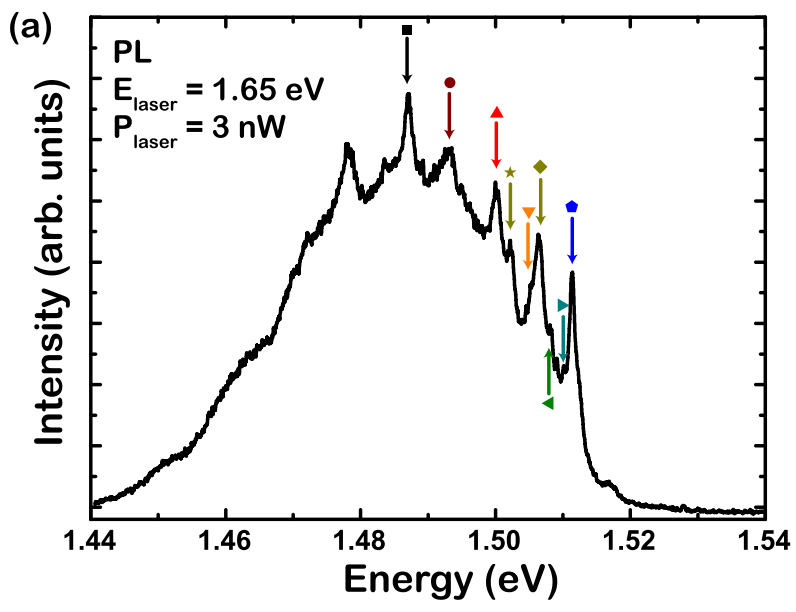

(c)

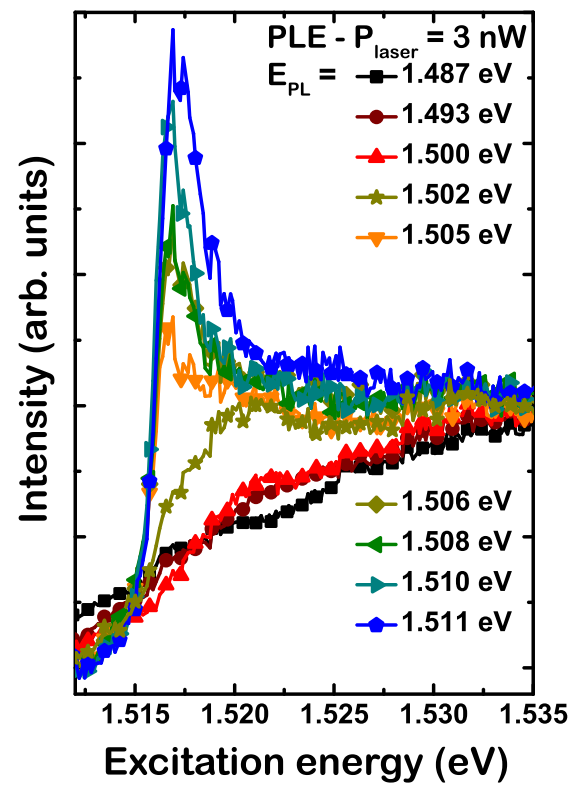

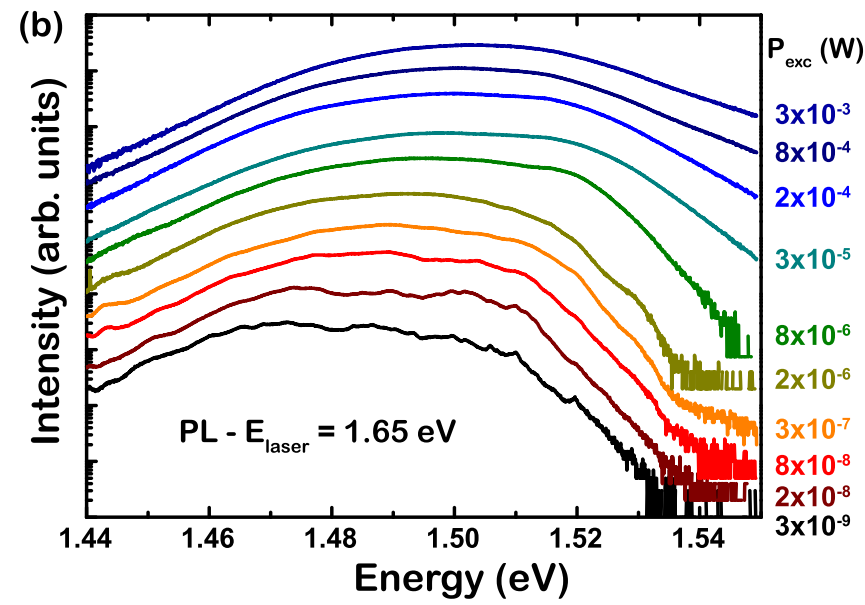

(e)
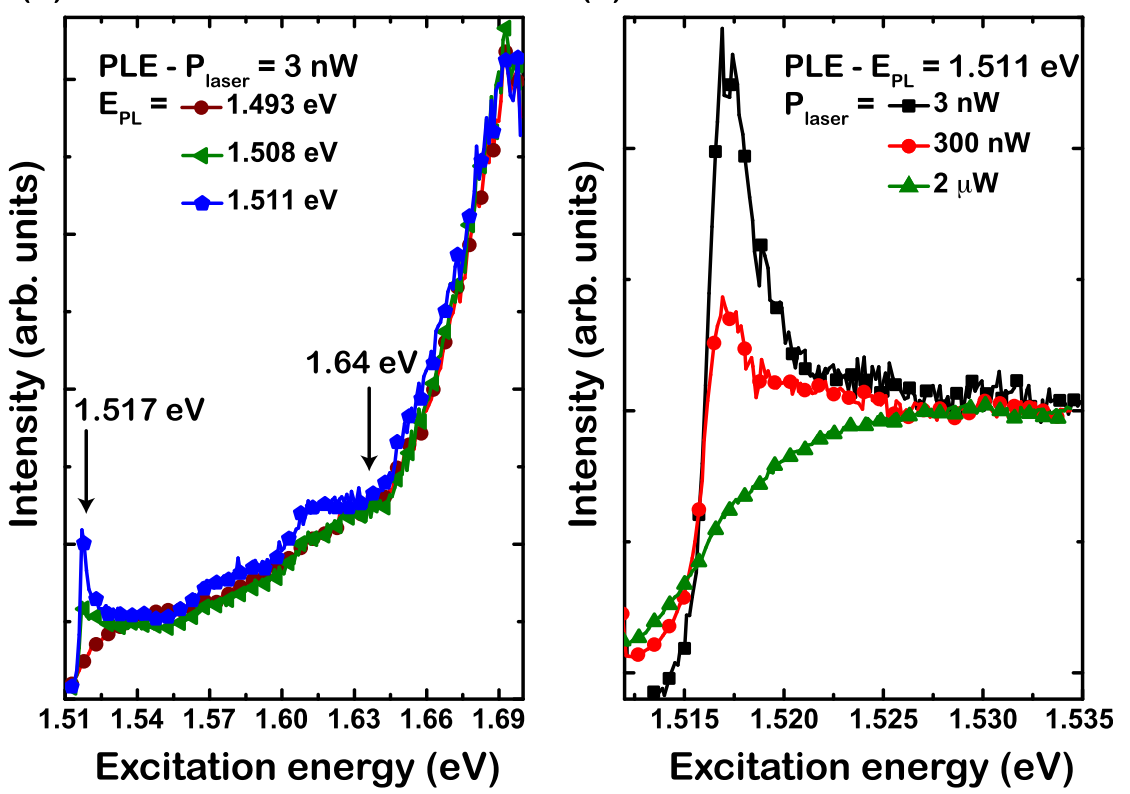

FIG. 1. (a) PL spectrum taken on an ensemble of as-grown nanowires with a laser excitation energy and power of 1.65 eV and 3 nW, respectively. Symbols show the detection energies for the PLE scans in (c) and (d). (b) PL spectra for excitation power between $3 \mathrm{nW}$ and $3 \mathrm{~mW}$ (from bottom to top). (c) and (d) PLE spectra taken with a laser excitation power of $3 \mathrm{nW}$ and a laser excitation energy between 1.512 and $1.535 \mathrm{eV}$ (c) and between 1.512 and $1.700 \mathrm{eV}$ (d). For clarity, PLE scans in (c) and (d) have been normalized to the emission intensity detected under laser excitation at 1.535 and 1.700 eV, respectively. (e) PLE scan of the $1.511 \mathrm{eV}$ emission taken with a laser excitation power of $3 \mathrm{nW}, 300 \mathrm{nW}$ and $2 \mu \mathrm{W}$ (black, red, and green lines, respectively). The PLE scans have been normalized to the emission intensity detected under laser excitation at $1.535 \mathrm{eV}$. 
(a)

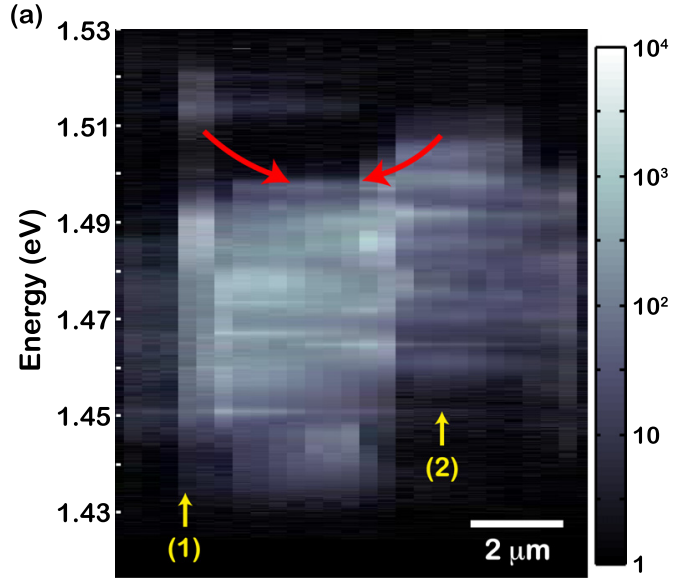

(b)

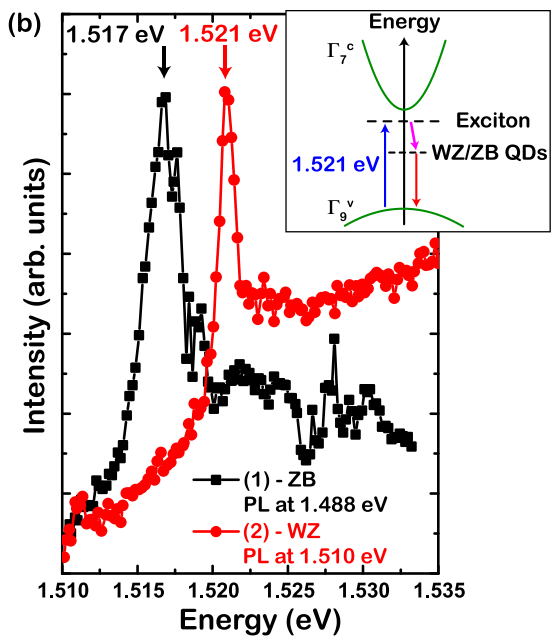

FIG. 2. (a) Micro-PL mapping taken along the length of a single GaAs nanowire. Red arrows highlight the redshift of the PL when going from the extremities to the central segment of the nanowire. (1) and (2) indicate where the PLE scans displayed in (b) have been acquired. (b) Normalized PLE scans taken at positions (1) and (2) (black and red spectra, respectively). Inset: Schematic band structure of WZ GaAs. The energy of the $\Gamma_{9}{ }^{\mathrm{v}}$ valence band to $\Gamma_{7}^{\mathrm{c}}$ conduction band exciton of wurtzite GaAs is $1.521 \mathrm{eV}$ at $4 \mathrm{~K}$. After excitation at $1.521 \mathrm{eV}$, excitons are generated resonantly in $\mathrm{WZ}$ GaAs segments (blue arrow), they diffuse and get trapped by $\mathrm{ZB}$ quantum discs (QDs) (purple arrow), where they recombine radiatively (red arrow). emission from the quantum discs at the extremities of the wire is 1.490 and $1.495 \mathrm{eV}$ (spectra (1) and (2) in Figure 2(a), respectively), but the central segment of the nanowire shows a PL peak between 1.460 and $1.480 \mathrm{eV}$. The emission energy is correlated with the local twinning and stacking fault density. ${ }^{17}$ In particular, where the local fault density is high, coupling between successive crystal-phase quantum discs leads to a decrease of the exciton energy of the quantum disc. ${ }^{31}$ In agreement with the transmission electron microscopy measurements performed on similar nanowires, ${ }^{17}$ the micro-PL scan in Figure 2(a) confirms the model that the two tips of our wires show ZB and WZ crystal structures with low twinning and stacking fault densities, respectively, and the central segments of the wires exhibit repeated alternation between $\mathrm{WZ}$ and $\mathrm{ZB}$ crystal structures.

We display in Figures 1(c) and 1(d) PLE scans taken with an excitation power of $3 \mathrm{nW}$ on $\mathrm{WZ} / \mathrm{ZB}$ quantum discs emitting between 1.487 and $1.511 \mathrm{eV}$. As the laser energy is scanned from 1.512 to $1.535 \mathrm{eV}$, we detect a strong enhancement of the $1.511 \mathrm{eV}$ emission when exciting at $1.517 \mathrm{eV}$ (Figure 1(c)). Since the nanowires studied show an equivalent volume fraction of ZB crystal phase larger than $80 \%$, we attribute the increase in emission intensity for excitation at $1.517 \mathrm{eV}$ to the resonant generation of heavy-hole excitons in $\mathrm{ZB}$ GaAs, followed by their trapping in the quantum discs. The $3 \mathrm{meV}$ inhomogeneous broadening of the heavy-hole exciton is the result of local variations of the strain state of the $\mathrm{ZB}$ phase along the length of the wire. ${ }^{32}$ We note that even when exciting the nanowire ensemble with a laser excitation energy below $1.516 \mathrm{eV}$, we detect emission from the quantum discs. This is evidence of light absorption by the $\mathrm{WZ} / \mathrm{ZB}$ discs. However, this process is inefficient, as only a weak PL signal is detected in this case (Figure 1(c)). When going from an excitation energy of 1.530 to $1.640 \mathrm{eV}$, the PL intensity at $1.511 \mathrm{eV}$ increases by a factor of $\sim 2.5$ (Figure 1(d)). Then, for higher laser energy, we observe an abrupt increase in emission intensity, which we attribute to the onset of absorption in the $\mathrm{Al}_{0.33} \mathrm{Ga}_{0.67} \mathrm{As}$ shells. ${ }^{33}$ All discs, irrespective of their emission energy, exhibit a similar PLE profile for laser energy larger than $1.530 \mathrm{eV}$ (Figure 1(d)), but this is not the case for excitation energy in the $1.512-1.530 \mathrm{eV}$ range. As shown in Figure 1(c), the lower the PL energy of the disc, the less pronounced the heavy-hole exciton resonance at $1.517 \mathrm{eV}$. We will demonstrate in the following that the disappearance of the exciton resonance in the PLE scans taken on low-energy emitting quantum discs is a direct consequence of the microstructure of our nanowires.

As discussed above, after excitation at $1.517 \mathrm{eV}$, excitons are generated resonantly in the $\mathrm{ZB} \mathrm{GaAs}$ segments with low stacking fault density. Conversely, the longer the $\mathrm{ZB}$ segment and the lower the local stacking fault density, the larger the nanowire absorption cross-section at $1.517 \mathrm{eV}$. At low excitation density, we observe almost no PL signal at $1.516 \mathrm{eV}$ (Figure 1(a)). Excitons generated in the ZB segments therefore diffuse (the exciton diffusion length in undoped GaAs can be of the order of a $\mu \mathrm{m}^{34}$ ) and get trapped by the nearby quantum discs, where they recombine radiatively. In agreement with the micro-PL scan in Figure 2(a), WZ quantum discs in nearly fault-free ZB GaAs segments give rise to the emission in the higher-energy part of the spectrum in Figure 1(a). Consequently, only discs emitting at higher energy may exhibit an increase in PL intensity due to resonant excitation at $1.517 \mathrm{eV}$, which is in agreement with the data in Figure 1(c). The evolution of the PLE spectrum of the quantum disc emitting at $1.511 \mathrm{eV}$ as a function of the excitation power corroborates this picture. As depicted in Figure 1(e), increasing the excitation density leads (i) to an increase of the PL signal when exciting below $1.516 \mathrm{eV}$ and (ii) to a disappearance of the excitonic resonance at $1.517 \mathrm{eV}$. We underline that even for the higher excitation power used here (i.e., $2 \mu \mathrm{W}$ ), our excitation conditions remain below the Mott transition for excitons. ${ }^{35}$ As a matter of fact, strong excitation leads to filling of the available WZ/ZB discs states (Figure 1(b)) even for excitation below $1.516 \mathrm{eV}$. As a result of this filling, the transfer of excitons from the $\mathrm{ZB}$ GaAs segments to the discs is hindered and no clear resonance is observed when exciting at $1.517 \mathrm{eV}$ (Figure 1(e)). Instead, carriers recombine radiatively in the fault-free $\mathrm{ZB}$ segments, giving rise to the $1.516 \mathrm{eV}$ emission band (Figure 1(b)).

We show in Figure 2(b) PLE scans taken at the two extremities of the nanowire in Figure 2(a) for an excitation energy between 1.512 and $1.535 \mathrm{eV}$. When exciting the lefthand extremity of the wire (position (1) in Figure 2(a)), the disc emission is maximum for a laser energy of $1.517 \mathrm{eV}$ (Figure 2(b)). In agreement with the data obtained on the 
wire ensemble (Figure 1), we deduce that the crystal structure of this wire segment consists of faulted ZB. When carrying out a PLE scan at the opposite tip of the wire (position (2) in Figure 2(a)), which exhibits accordingly a faulted WZ structure, we detect a resonance centred at $1.521 \mathrm{eV}$ with a $2 \mathrm{meV}$ linewidth. This resonance arises from the resonant generation of excitons in WZ GaAs followed by their trapping into the nearby $\mathrm{ZB}$ quantum discs. The fundamental conduction band of usual semiconductor compounds with WZ crystal structure, such as a $\mathrm{GaN}$ or $\mathrm{ZnO}$, is $\Gamma_{7}^{\mathrm{c}}, 36$ $a b$ initio calculations have predicted either a $\Gamma_{8}{ }^{\mathrm{c}}$ (Ref. 11) or a $\Gamma_{7}{ }^{\mathrm{c}}$ symmetry ${ }^{37}$ for the lowest-energy conduction band of WZ GaAs. Although both the transitions from $\Gamma_{7}{ }^{\mathrm{c}}$ and $\Gamma_{8}{ }^{\mathrm{c}}$ to the heavy-hole valence band $\Gamma_{9}{ }^{\mathrm{v}}$ are allowed for light polarized perpendicular to the wire axis, ${ }^{38}$ in practice the oscillator strength of the $\Gamma_{8}{ }^{\mathrm{c}} \rightarrow \Gamma_{9}{ }^{\mathrm{v}}$ transition is almost zero. ${ }^{37}$ Consequently, we attribute the exciton transition at $1.521 \mathrm{eV}$ to $\Gamma_{7}{ }^{\mathrm{c}} \rightarrow \Gamma_{9}^{\mathrm{v}}$, in agreement with the RRS experiments on a single nanowire reported in Ref. 15 . We note that the line broadening observed here for the $\Gamma_{7}^{\mathrm{c}} \rightarrow \Gamma_{9}^{\mathrm{v}}$ transition is $2 \mathrm{meV}$, to be compared with the $8 \mathrm{meV}$ extracted by RRS in Ref. 15. It is at first surprising that the exciton linewidth obtained from a coherent technique is larger than what we observe by PLE. However, the excitation density used for the experiments in Figure 2(b) $\left(0.17 \mathrm{~W} / \mathrm{cm}^{2}\right)$ is lower than what is used usually for RRS and we speculate that the difference in exciton linewidth originates from a powerinduced broadening of the exciton transition. Finally, we would like to mention that excitons bound to GaAs WZ/ZB quantum discs exhibit scattered values of diamagnetic coefficient ${ }^{23}$ and a large scattering rate with acoustic phonons. ${ }^{22}$ While $\Gamma_{7}^{\mathrm{c}}$ electrons are expected to show an effective mass similar to that of the $\Gamma_{6}{ }^{\mathrm{c}}$ conduction band of $\mathrm{ZB} \mathrm{GaAs},{ }^{11,37}$ the reports in Refs. 22 and 23 suggest rather that the electron mass is heavy. Since the $\Gamma_{7}{ }^{\mathrm{c}}$ and $\Gamma_{8}{ }^{\mathrm{c}}$ states show a light and heavy electron mass, respectively, ${ }^{11,36}$ we propose that electronic confinement in WZ/ZB GaAs crystal-phase quantum discs leads to a heavy-electron light-electron mixing, in a way similar to the heavy-hole light-hole mixing reported in Ref. 39. The resulting conduction state would exhibit a high effective mass as well as a non-zero oscillator strength for recombination to the valence band, in agreement with experimental reports. For such a mixing to be possible, the energy separation between the $\Gamma_{7}^{\mathrm{c}}$ and $\Gamma_{8}^{\mathrm{c}}$ conduction bands must be small. This agrees qualitatively with theoretical calculations of the band structure of WZ GaAs, which predict systematically that the energy difference between the $\Gamma_{7}^{\mathrm{c}}$ and $\Gamma_{8}{ }^{\mathrm{c}}$ bands is smaller than $85 \mathrm{meV} .{ }^{11,17,37}$

In conclusion, we have performed PL and PLE experiments on polytypic GaAs nanowires both individually and in ensembles. At $4 \mathrm{~K}$, the nanowire PL is dominated by the recombination of excitons bound to WZ/ZB quantum discs. The PLE of ensembles of quantum discs shows a strong resonance at $1.517 \mathrm{eV}$, as a result of resonant generation of heavy-hole excitons in the ZB GaAs segments followed by their trapping by the discs. For higher excitation density, filling of the discs states results in the disappearance of the $1.517 \mathrm{eV}$ resonance. Instead, we observe a higher-energy emission band centred at $1.516 \mathrm{eV}$ that we attribute to the recombination of carriers in nearly fault-free ZB GaAs wire segments. We have then deduced from micro-PLE scans taken along the length of single nanowires that the energy of the $\Gamma_{7}{ }^{\mathrm{c}} \rightarrow \Gamma_{9}{ }^{\mathrm{v}}$ exciton in WZ GaAs is $1.521 \mathrm{eV}$ at $4 \mathrm{~K}$. Coupled with previous reports, these results suggest that the lowest energy electron state in GaAs crystal-phase quantum discs results from the mixing between the $\Gamma_{7}^{\mathrm{c}}$ and $\Gamma_{8}^{\mathrm{c}}$ conduction bands of WZ GaAs.

We acknowledge funding from the European Seventh Framework Programme under grant agreement 265073 and from the Hitachi Cambridge Laboratory. A.F.i.M. and E.U. acknowledge funding from the Marie Curie Excellence Grant SENFED.

${ }^{1}$ J. Wallentin, N. Anttu, D. Asoli, M. Huffman, I. Aberg, M. H. Magnusson, G. Siefer, P. Fuss-Kailuweit, F. Dimroth, B. Wizigmann, K. Deppert, and M. T. Borgstrom, Science 339, 1057 (2013).

${ }^{2}$ P. Krogstrup, H. I. Jorgensen, M. Heiss, O. Demichel, J. V. Holm, M. Aagesen, J. Nygard, and A. Fontcuberta i Morral, Nat. Photonics 7, 306 (2013).

${ }^{3}$ T. M. Babinec, B. J. M. Hausmann, M. Khan, Y. Zhang, J. R. Maze, P. R. Hemmer, and M. Loncar, Nat. Nanotechnol. 5, 195 (2010).

${ }^{4}$ J. Claudon, J. Bleuse, N. S. Malik, M. Bazin, P. Jaffrenou, N. Gregersen, C. Sauvan, P. Lalanne, and J. M. Gérard, Nat. Photonics 4, 174 (2010).

${ }^{5}$ O. Gazzano, S. Michaelis de Vasconcellos, C. Arnold, A. Nowak, E. Galopin, I. Sagnes, L. Lanco, A. Lemaître, and P. Senellart, Nat. Commun. 4, 1425 (2013).

${ }^{6}$ M. Heiss, Y. Fontana, A. Gustafsson, G. Wüst, C. Magen, D. D. O’Regan, J. W. Luo, B. Ketterer, S. Conesa-Boj, A. V. Kuhlmann, J. Houel, E. RussoAverchi, J. R. Morante, M. Cantoni, N. Marzari, J. Arbiol, A. Zunger, R. J. Warburton, and A. Fontcuberta i Morral, Nature Mater. 12, 439 (2013).

${ }^{7}$ M. E. Reimer, G. Bulgarini, N. Akopian, M. Hocevar, M. B. Bavinck, M. A. Verheijen, E. P. A. M. Bakkers, L. P. Kouwenhoven, and V. Zwiller, Nat. Commun. 3, 737 (2012).

${ }^{8}$ A. Tribu, G. Sallen, T. Aichele, R. André, J.-P. Poizat, C. Bougerol, S. Tatarenko, and K. Kheng, Nano Lett. 8, 4326 (2008).

${ }^{9}$ D. Bajoni, P. Senellart, E. Wertz, I. Sagnes, A. Miard, A. Lemaitre, and J. Bloch, Phys. Rev. Lett. 100, 047401 (2008).

${ }^{10}$ A. Das, J. Heo, M. Jankowski, W. Guo, L. Zhang, H. Deng, and P. Bhattacharya, Phys. Rev. Lett. 107, 066405 (2011).

${ }^{11}$ A. De and C. E. Pryor, Phys. Rev. B 81, 155210 (2010).

${ }^{12}$ P. Caroff, K. Dick, J. Johansson, M. E. Messing, K. Deppert, and L. Samuelson, Nat. Nanotechnol. 4, 50 (2009).

${ }^{13}$ N. Akopian, G. Patriarche, L. Liu, J.-C. Harmand, and V. Zwiller, Nano Lett. 10, 1198 (2010).

${ }^{14}$ H. L. Zhou, T. B. Hoang, D. L. Dheeraj, A. T. J. van Helvoort, L. Liu, J. C. Harmand, B. O. Fimland, and H. Weman, Nanotechnology 20, 415701 (2009).

${ }^{15}$ B. Ketterer, M. Heiss, E. Uccelli, J. Arbiol, and A. Fontcuberta i Morral, ACS Nano 5, 7585 (2011).

${ }^{16}$ P. Kusch, S. Breuer, M. Ramsteiner, L. Geelhaar, H. Riechert, and S. Reich, Phys. Rev. B 86, 075317 (2012).

${ }^{17}$ M. Heiss, S. Conesa-Boj, J. Ren, H.-H. Tseng, A. Gali, A. Rudolph, E. Uccelli, F. Peiro, J. R. Morante, D. Schuh, E. Reiger, E. Kaxiras, J. Arbiol, and A. Fontcuberta i Morral, Phys. Rev. B 83, 045303 (2011).

${ }^{18}$ L. Ahtapodov, J. Todorovic, P. Olk, T. Mjåland, P. Slåttnes, D. L. Dheeraj, A. T. J. van Helvoort, B.-O. Fimland, and H. Weman, Nano Lett. 12, 6090 (2012).

${ }^{19}$ U. Jahn, J. Lähnemann, C. Pfüller, O. Brandt, S. Breuer, B. Jenichen, M. Ramsteiner, L. Geelhaar, and H. Riechert, Phys. Rev. B 85, 045323 (2012).

${ }^{20}$ T. B. Hoang, A. F. Moses, H. L. Zhou, D. L. Dheeraj, B. O. Fimland, and H. Weman, Appl. Phys. Lett. 94, 133105 (2009).

${ }^{21}$ T. Cheiwchanchamnangij and W. R. L. Lambrecht, Phys. Rev. B 84, 035203 (2011).

${ }^{22}$ A. M. Graham, P. Corfdir, M. Heiss, S. Conesa-Boj, E. Uccelli, A. Fontcuberta i Morral, and R. T. Phillips, Phys. Rev. B 87, 125304 (2013).

${ }^{23}$ P. Corfdir, B. Van Hattem, E. Uccelli, S. Conesa-Boj, P. Lefebvre, A. Fontcuberta i Morral, and R. T. Phillips, "Three-dimensional magnetophotoluminescence as a probe of the electronic properties of crystal-phase quantum disks in GaAs nanowires," Nano Lett. (submitted).

${ }^{24}$ W. Peng, F. Jabeen, B. Jusserand, J. C. Harmand, and M. Bernard, Appl. Phys. Lett. 100, 073102 (2012). 
${ }^{25}$ P. Corfdir, P. Lefebvre, J. Ristić, P. Valvin, E. Calleja, A. Trampert, J.-D. Ganière, and B. Deveaud-Plédran, J. Appl. Phys. 105, 013113 (2009).

${ }^{26}$ O. Brandt, C. Pfüller, C. Chèze, L. Geelhaar, and H. Riechert, Phys. Rev. B 81, 045302 (2010).

${ }^{27}$ P. Corfdir, M. Abid, A. Mouti, P. A. Stadelmann, E. Papa, J.-P. Ansermet, J.-D. Ganière, and B. Deveaud-Plédran, Nanotechnology 22, 285710 (2011).

${ }^{28}$ S. Perera, K. Pemasiri, M. A. Fickenscher, H. E. Jackson, L. M. Smith, J. Yarrison-Rice, S. Paiman, Q. Gao, H. H. Tan, and C. Jagadish, Appl. Phys. Lett. 97, 023106 (2010).

${ }^{29}$ G. Jacopin, L. Rigutti, L. Largeau, F. Fortuna, F. Furtmayr, F. H. Julien, M. Eickhoff, and M. Tchernycheva, J. Appl. Phys. 110, 064313 (2011).

${ }^{30}$ O. Demichel, M. Heiss, J. Bleuse, H. Mariette, and A. Fontcuberta i Morral, Appl. Phys. Lett. 97, 201907 (2010).

${ }^{31}$ P. Corfdir and P. Lefebvre, J. Appl. Phys. 112, 053512 (2012).
${ }^{32}$ I. Zardo, S. Conesa-Boj, F. Peiro, J. R. Morante, J. Arbiol, E. Uccelli, G. Abstreiter, and A. Fontcuberta i Morral, Phys. Rev. B 80, 245324 (2009).

${ }^{33}$ B. Ketterer, M. Heiss, M. J. Livrozet, A. Rudolph, E. Reiger, and A. Fontcuberta i Morral, Phys. Rev. B 83, 125307 (2011).

${ }^{34}$ D. Araújo, G. Oelgart, J.-D. Ganière, and F. K. Reinhart, Appl. Phys. Lett. 62, 2992 (1993).

${ }^{35}$ C. K. Yong, H. J. Joyce, J. Lloyd-Hugues, Q. Gao, H. H. Tan, C. Jagadish, M. B. Johnston, and L. M. Herz, Small 8, 1725 (2012).

${ }^{36}$ B. Gil and O. Briot, Phys. Rev. B 55, 2530 (1997); B. Gil, Phys. Rev. B 64, 201310(R) (2001).

${ }^{37}$ F. Bechstedt and A. Belabbes, J. Phys.: Condens. Matter 25, 273201 (2013).

${ }^{38}$ P. Tronc, Yu. E. Kitaev, G. Wang, M. F. Limonov, A. G. Panfilov, and G. Neu, Phys. Status Solidi B 216, 599 (1999).

${ }^{39}$ D. Spirkoska, Al. L. Efros, W. R. L. Lambrecht, T. Cheiwchanchamnangij, A. Fontcuberta i Morral, and G. Abstreiter, Phys. Rev. B 85, 045309 (2012). 